\title{
Analysis and Experiment of Helical Spring-Type Pressure and Shear Sensor *
}

\author{
Naoyuki TAKESUE**, Akihito SANO**, Hiromi MOCHIYAMA**, \\ Ryo KIKUUWE ${ }^{* *}$, Taiki ISHIGURO** and Hideo FUJIMOTO** \\ ** Nagoya Institute of Technology \\ Gokiso-cho, Showa-ku, Nagoya-shi, Aichi, 466-8555 Japan \\ E-mail: takesue@nitech.ac.jp
}

\begin{abstract}
In this paper, we propose a sensor that detects both pressure and shear information by a helical spring and strain gages. The stresses working on the wire of spring are analyzed. The guideline to attach strain gages on the spring is discussed and the calibration procedure is shown. Finally, experiments are carried out and the effectiveness of the sensor is shown.
\end{abstract}

Key words : Touch Sensation, Tactile Sensor, Pressure, Shear, Strain Gage, Helical Spring

\section{Introduction}

Tactile sensing is an attractive field in robotics. So far, the tactile sensors have been studied by many researchers. When an object is grasped and lifted, the compressive force is an important information, and the shearing force also plays an important role. The mechanism of the perception have been studied ${ }^{(1),(2)}$.

The slip sensor using PVDF film ${ }^{(3)}$ has been proposed as a tactile sensor detecting the friction coefficient in case of grasping an object. In the human skin, the mechanoreceptor that is said to be responsive to the shearing force is Meissner's corpuscle, which is helical shaped, about $150[\mu \mathrm{m}]$ in length and $40 \sim 70[\mu \mathrm{m}]$ in diameter. Nara et al. ${ }^{(4)}$ have mathematically derived the properties of Meissner's corpuscle and others, and have indicated the possibility of the application based on the principle. The helical coil plays a role to translate the extension and compression/shearing deformation of the whole coil to the shearing/longitudinal deformation on the surface of the coil wire. Sano et al. ${ }^{(5)}$ have developed the soft fingers in which tiny coil springs with strain gages were arranged into silicone resins with different hardness, and have succeeded in detecting the slipping condition of handling an object by using it. One of the advantages for the use of coil spring is that it allows a large deformation.

In this paper, we analytically explain the principle of the helical spring-type shearing sensor developed by Sano et al. ${ }^{(5)}$ and show the method to detect the compressive information at the same time in addition to the shearing information. The moments working to a coil spring have been generally derived ${ }^{(6)}$. It is, however, for the design of the spring as a mechanical component. No example that uses a helical spring with strain gage as a sensor has been seen. The present paper derives the guideline for the appropriate angle of the strain gage for the pressure and shear sensor based on the moments working to the helical spring, and shows the calibration procedure using least square approximation. Finally, experiments are carried out and the effectiveness of sensor is shown.

\section{Helical spring-type pressure and shear sensor}

\subsection{Sensor concept}

The sensor concept is shown in Fig. 1. The $x$-axis is a tangent direction to the wire of spring on which strain gage is attached, the $y$-axis is horizontal and perpendicular to $x$-axis and the $z$-axis is the vertical direction, namely axis of a helical spring. 

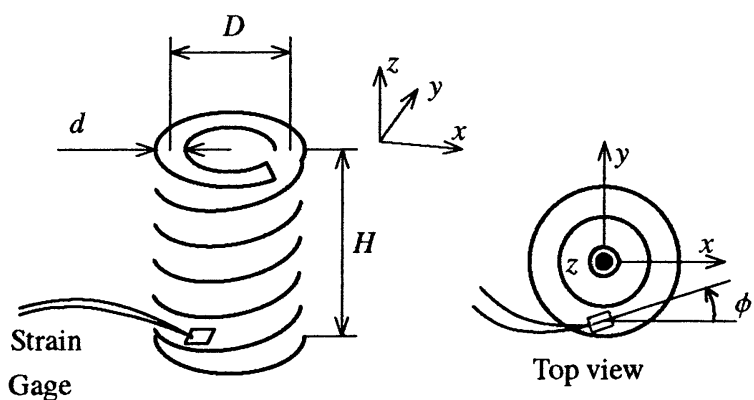

Top view

Fig. 1 Concept chart of helical spring-type pressure and shear sensor
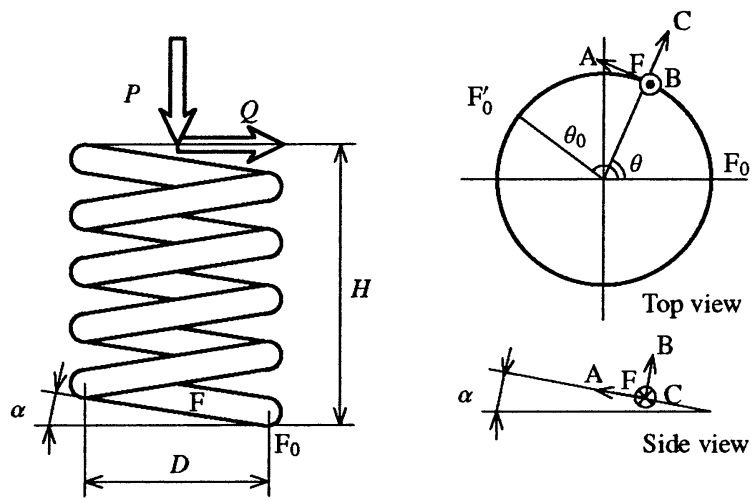

Fig. 2 Moments working to helical spring

When the helical spring is compressed (z-deformation), the torsional moment is mainly caused to the spring wire. On the other hand, when the spring is sheared to $x$-direction, the bending moment is mainly caused to the wire on which the gage is attached, however, $y$-direction shear doesn't cause the bending moment.

Selective detection of pressure and shear deformation is possible by attaching strain gages to a helical spring and detecting the torsional and bending moments. The detail explanation is shown in the following sections.

\subsection{Moments on wire of spring}

The vector chart of the moments affecting a helical spring is shown in Fig. $2^{(6)}$. The definition of symbols is shown in Table 1.

Table 1 Definition of symbols

\begin{tabular}{c|l}
\hline Symbol & Definition \\
\hline$D$ & diameter of spring \\
$R$ & radius of spring $(=D / 2)$ \\
$H$ & height of spring \\
$\alpha$ & pitch angle \\
$P$ & pressure load \\
$Q$ & shear load \\
$F_{0}$ & beginning point of spring \\
$F_{0}^{\prime}$ & ending point of spring \\
$F$ & point of element \\
$\theta_{0}$ & position of ending point \\
$\theta$ & position of element \\
\hline
\end{tabular}

A compressive force (axial load), $P$, and a shearing force (lateral load), $Q$, is given on the top of the helical spring as shown in Fig. 2. In this circumstance, omitting the constrain force on the end of spring, the total moments, $M_{1}, M_{2}$ and $M_{3}$, around FA, FB and FC axes 
are written as follows, respectively ${ }^{(6)}$ :

$$
\begin{aligned}
& M_{1}=-P R \cos \alpha+Q\left[H\left(1-\frac{\theta}{\theta_{0}}\right) \cos \theta \cos \alpha+R \sin \theta \sin \alpha\right], \\
& M_{2}=P R \sin \alpha-Q\left[H\left(1-\frac{\theta}{\theta_{0}}\right) \cos \theta \sin \alpha+R \sin \theta \cos \alpha\right], \\
& M_{3}=Q H\left(1-\frac{\theta}{\theta_{0}}\right) \sin \theta .
\end{aligned}
$$

When $\theta_{0}=2 n \pi$ and $\theta=\pi / 2$, which mean that FA-direction and the shear direction are the same, each moment is written as follows:

$$
\begin{aligned}
& M_{1}=-P R \cos \alpha+Q R \sin \alpha, \\
& M_{2}=P R \sin \alpha-Q R \cos \alpha, \\
& M_{3}=\frac{4 n-1}{4 n} Q H .
\end{aligned}
$$

For simplicity, assuming that the pitch angle of spring, $\alpha$, is as small as $\sin \alpha \simeq 0, \cos \alpha \simeq 1$ and that the number of turns, $n$, is as large as $\frac{4 n-1}{4 n} \simeq 1$, the equations are expressed as follows:

$$
\begin{aligned}
& M_{1}=-P R, \\
& M_{2}=-Q R, \\
& M_{3}=Q H .
\end{aligned}
$$

Thus, if the shear direction is the same with FA-direction, the compressive force, $P$, can be detected from the torsional moment, $M_{1}$, and the shearing force, $Q$, from the bending moment, $M_{3}$ or $M_{2}$.

\subsection{Stresses and strains}

Assuming that the diameter of wire is enough small comparing with the diameter of coil, the strains on the inner and outer surfaces of wire are considered to be almost the same.

The torsional (shearing) stress, $\tau$, on the wire is written with the torsional moment, $M_{1}$, as follows:

$$
\tau=\frac{M_{1}}{2 Z}
$$

where $Z$ is a modulus of section.

Therefore, the compressive force, $P$, can be obtained when a strain gage is attached on the wire of spring and the shearing strain, $\gamma$, caused by shearing stress, $\tau$, is measured.

$$
\gamma=\frac{\tau}{G}=-\frac{P R}{2 G Z}
$$

The longitudinal stress as shown in the following equation is caused on the upper and lower parts of the wire by the bending moment.

$$
\sigma=\mp \frac{M_{3}}{Z}
$$

where $Z$ is a modulus of section.

By detecting the longitudinal strain, $\varepsilon$, caused by the bending moment, the shearing force, $Q$, can be obtained.

$$
\varepsilon=\frac{\sigma}{E}=\frac{Q H}{E Z} .
$$




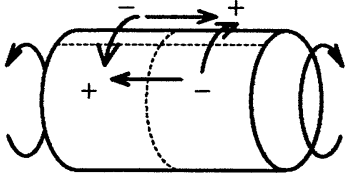

Upper side

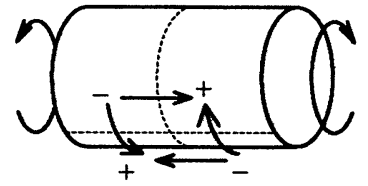

Lower side

Torsional moment

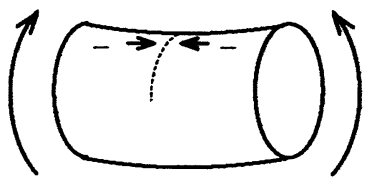

Upper side

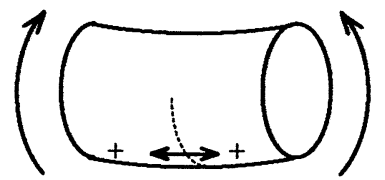

Lower side

Bending moment

Fig. 3 Torsional and bending moments on wire

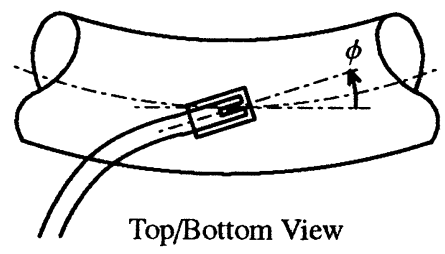

Fig. 4 Angle of gage on wire

\subsection{Separation of torsional and bending signals}

When compression and shear affect a spring, the torsional and bending moments shown in Fig. 3 are caused.

On the upper and lower surfaces of wire, the stresses in direction of $\phi$ from the axial direction of wire are expressed as follows:

$$
\begin{aligned}
& \sigma_{\mathrm{I}}=-\frac{\sigma}{2}-\frac{1}{2} \sqrt{\sigma^{2}+4 \tau^{2}} \cos 2(\psi-\phi), \\
& \sigma_{\mathrm{II}}=\frac{\sigma}{2}+\frac{1}{2} \sqrt{\sigma^{2}+4 \tau^{2}} \cos 2(\psi+\phi),
\end{aligned}
$$

where $\sigma_{\mathrm{I}}$ is the stress on the upper surface, and $\sigma_{\mathrm{II}}$ is the one on the lower. $\psi$ is an angle of principal stress and is expressed with torsional and bending stresses, $\tau$ and $\sigma$, as the following equation:

$$
\tan 2 \psi=\frac{2 \tau}{\sigma}
$$

The circle of stress is shown in Fig. 5.

The sum and difference of stresses $\sigma_{\mathrm{I}}$ and $\sigma_{\mathrm{II}}$ are written as follows:

$$
\begin{aligned}
& S_{p}=\sigma_{\mathrm{I}}+\sigma_{\mathrm{II}}=-2 \tau \sin 2 \phi, \\
& S_{s}=\sigma_{\mathrm{I}}-\sigma_{\mathrm{II}}=-\sigma(1+\cos 2 \phi) .
\end{aligned}
$$

Therefore, the torsional and bending stresses, $\tau$ and $\sigma$, can be separated from the original stresses, $\sigma_{\mathrm{I}}$ and $\sigma_{\mathrm{II}}$.

When $\phi=0, S_{p}$ anytime becomes zero and the torsional stress, $\tau$, cannot be detected. This means that the angle should be $\phi>0$. On the other hand, when $\phi=\pi / 2$, the bending stress, $\sigma$, cannot be obtained. In order to measure both torsional and bending stresses, the angle should be $0<\phi<\pi / 2$. 


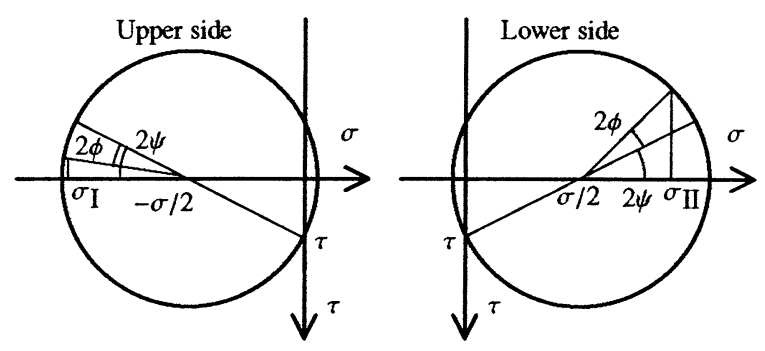

Fig. 5 Circle of stress

\subsection{Discussion on error of angle}

When a strain gage is attached on a wire, an error of the angle must be caused. In this section, the influence of angular error to measurement is considered. From Eqs. (14) and (15), the following equations are obtained.

$$
\begin{aligned}
& \frac{\partial \sigma_{\mathrm{I}}}{\partial \phi}=-\sqrt{\sigma^{2}+4 \tau^{2}} \sin 2(\psi-\phi), \\
& \frac{\partial \sigma_{\mathrm{II}}}{\partial \phi}=-\sqrt{\sigma^{2}+4 \tau^{2}} \sin 2(\psi+\phi) .
\end{aligned}
$$

The difference between the angles of the upper and lower gages is defined to be $\delta \phi$, and the average of the angles to be $\phi$. Then, the errors $\delta S_{p}$ and $\delta S_{s}$ caused on $S_{p}$ and $S_{s}$ are as follows:

$$
\begin{aligned}
\delta S_{p} & =\frac{\partial \sigma_{\mathrm{I}}}{\partial \phi} \frac{\delta \phi}{2}+\frac{\partial \sigma_{\mathrm{II}}}{\partial \phi} \frac{-\delta \phi}{2} \\
& =\left(\sqrt{\sigma^{2}+4 \tau^{2}} \cos 2 \psi \sin 2 \phi\right) \delta \phi \\
& =(\sigma \sin 2 \phi) \delta \phi, \\
\delta S_{s} & =\frac{\partial \sigma_{\mathrm{I}}}{\partial \phi} \frac{\delta \phi}{2}-\frac{\partial \sigma_{\mathrm{II}}}{\partial \phi} \frac{-\delta \phi}{2} \\
& =\left(-\sqrt{\sigma^{2}+4 \tau^{2}} \sin 2 \psi \cos 2 \phi\right) \delta \phi \\
& =(-2 \tau \cos 2 \phi) \delta \phi .
\end{aligned}
$$

The ratios of the stress errors are obtained:

$$
\begin{aligned}
\frac{\delta S_{p}}{S_{p}} & =-\frac{\sigma}{2 \tau} \delta \phi, \\
\frac{\delta S_{s}}{S_{s}} & =-\frac{2 \tau \cos 2 \phi}{\sigma(1+\cos 2 \phi)} \delta \phi .
\end{aligned}
$$

From these equations, it can be seen that with respect to $S_{p}$ the influence of error, $\delta S_{p} / S_{p}$, is independent from $\phi$ and is constant, and with respect to $S_{s}$ the influence of error, $\delta S_{s} / S_{s}$, is minimum in the case of $\phi=\pi / 4$.

If the error $\delta \phi \ll 1$, the influence may be nonsignificant. However, if the error is not small enough, the slope of the signal shifts and the calibration is needed.

\subsection{Guideline for angle of strain gage}

The guideline for the angle of the strain gage on the spring wire is summarized from the results shown in $\S 2.4$ and 2.5 .

First, the gages on the upper and lower surfaces should be inclined to the same direction, since it is difficult to separate the signal to torsional and bending components if the gages are attached at the different angles such as $\pm \phi$.

Second, it should be $\phi \neq 0$ to detect the torsional deformation, and should be $\phi \neq \pi / 2$ to detect the bending one.

It seems that $\phi=\pi / 4$ is the best angle in order to suppress the influence of the error of the gages for the bending deformation. In case of $\phi=\pi / 4$, however, the sensitivity of torsional 
deformation tends to be higher than the one of bending. For instance, assuming that the ratio of the maximum of the torsional and bending stresses is $2 \tau_{\max }=\sigma_{\max }$, the angle of $\phi=\pi / 8$ is more appropriate in which $\left|S_{p}+S_{s}\right|$ becomes maximum as seen from Eq. (17) and Eq. (18).

\subsection{Calibration procedure from gage voltages to displacements}

The guideline for gage angles is shown in the previous section to detect the torsional and bending signals under the appropriate condition. Since the errors of gage itself, bridge resistance, and so on may influence the offsets and coefficients of the signals of gages, the calibration procedure from the gage voltages to compressive and shearing displacements of the spring is shown below.

It can be considered that the voltage of gage 1 (the upper side), $v_{1}$, is expressed as the following equation:

$$
v_{1}=k_{10}+k_{11} z+k_{12} x
$$

where $z$ is the displacement of the compression of the spring, and $x$ is the displacement of the shear. $k_{10}, k_{11}$ and $k_{12}$ are the coefficients to be obtained.

Similarly, the voltage of gage 2 (the lower side), $v_{2}$, is given as below:

$$
v_{2}=k_{20}+k_{21} z+k_{22} x \text {. }
$$

The gage voltages, $v_{1}$ and $v_{2}$, are experimentally measured when the displacements of the compression and share are given to the spring. And the coefficients $k_{10} \sim k_{22}$ are calculated using least square approximation from the experimental results.

$$
\left[\begin{array}{lll}
k_{10} & k_{11} & k_{12} \\
k_{20} & k_{21} & k_{22}
\end{array}\right]^{T}=A^{-1} B,
$$

where

$$
\begin{aligned}
A & =\left[\begin{array}{ccc}
N & \Sigma z & \Sigma x \\
\Sigma z & \Sigma z^{2} & \Sigma z x \\
\Sigma x & \Sigma z x & \Sigma x^{2}
\end{array}\right], \\
B & =\left[\begin{array}{ccc}
\Sigma v_{1} & \Sigma v_{1} z & \Sigma v_{1} x \\
\Sigma v_{2} & \Sigma v_{2} z & \Sigma v_{2} x
\end{array}\right]^{T} .
\end{aligned}
$$

The above equations (25) and (26) are summarized as below:

$$
\left[\begin{array}{l}
v_{1} \\
v_{2}
\end{array}\right]=\left[\begin{array}{l}
k_{10} \\
k_{20}
\end{array}\right]+\left[\begin{array}{ll}
k_{11} & k_{12} \\
k_{21} & k_{22}
\end{array}\right]\left[\begin{array}{l}
z \\
x
\end{array}\right] .
$$

Therefore, if the coefficients $k_{10} \sim k_{22}$ are obtained, the displacement $z$ and $x$ are calculated from the gage voltages $v_{1}$ and $v_{2}$ as the following equation:

$$
\left[\begin{array}{l}
z \\
x
\end{array}\right]=\left[\begin{array}{ll}
k_{11} & k_{12} \\
k_{21} & k_{22}
\end{array}\right]^{-1}\left(\left[\begin{array}{l}
v_{1} \\
v_{2}
\end{array}\right]-\left[\begin{array}{l}
k_{10} \\
k_{20}
\end{array}\right]\right) .
$$

\section{Experiments}

\subsection{Tested helical spring}

Strain gages are attached onto wire of a helical spring and the signals are measured in case of pressing and shearing of spring. The used strain gages are KFR-02N-120-C1-16 N10C2 made by Kyowa Electronic Instruments Co., Ltd. The size is $1.2[\mathrm{~mm}] \times 1.6[\mathrm{~mm}]$.

The helical spring of a rectangular wire is used because of the easiness of attaching gages. The size of helical spring is shown in Table 2.

The picture of the spring is shown in Fig. 6. 
Table 2 Specification of helical spring

\begin{tabular}{l|r}
\hline Diameter of spring: $D$ & $9[\mathrm{~mm}]$ \\
Height of spring: $H$ & $20[\mathrm{~mm}]$ \\
Width of wire: $a$ & $2[\mathrm{~mm}]$ \\
Thickness of wire: $b$ & $0.8[\mathrm{~mm}]$ \\
\hline
\end{tabular}

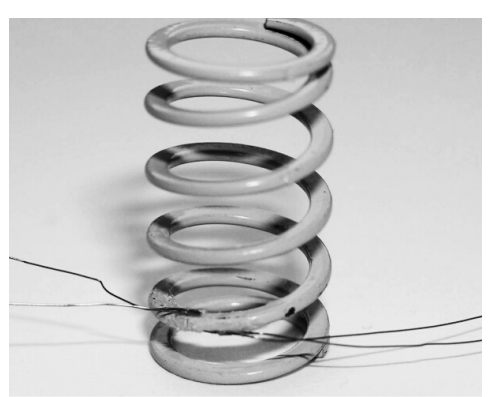

Fig. 6 Picture of helical spring with strain gages

\subsection{Experimental setup}

An experimental setup to compress and shear the spring with strain gages is shown in Fig. 7. The compressing and shearing displacements are given by micrometers and the voltages are measured through a Wheatstone bridge box.

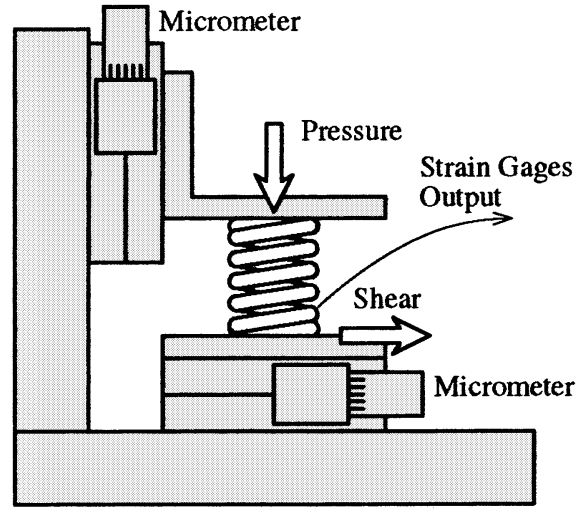

Fig. 7 Experimental setup

\subsection{Output signals}

First, the spring is compressed up to the displacement of $8[\mathrm{~mm}]$. Figure 8 shows the raw output voltages of gage 1 and gage 2 that are gages on the upper and lower surfaces, respectively.

Next, the pressure displacement is fixed to $2,4,6$ or 8 [mm], and the spring is sheared up to the displacement of $2[\mathrm{~mm}]$. The coefficients $k_{10} \sim k_{22}$ shown in $\S 2.7$ are calculated from the data using least square approximation.

$$
\begin{array}{lll}
k_{10}: 0.234 & k_{11}: 0.219 & k_{12}:-0.406 \\
k_{20}:-0.164 & k_{21}: 0.201 & k_{22}: 0.430
\end{array}
$$

The compression and shear components $(z$ and $x$ ) are obtained from the coefficients. The components obtained from Eq. (29) and the signals in case of compression (Fig. 8) are shown in Fig. 9. The components obtained from the signals in case of shearing are shown in Fig. 10. As seen from these figures, both results showed that the compression and shear components were well separated although some hysteresis are observed in shear component in Fig. 10. It may be because the upper and lower ends of spring are deviated from the original position in shearing. 


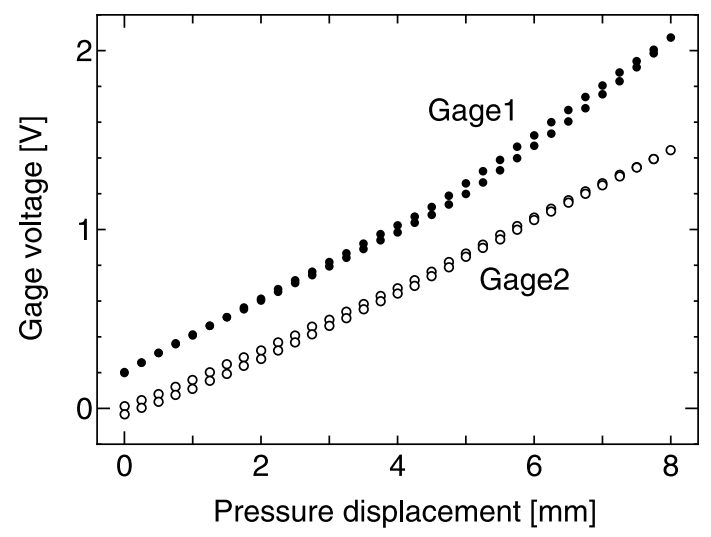

Fig. 8 Raw outputs of gages under compression

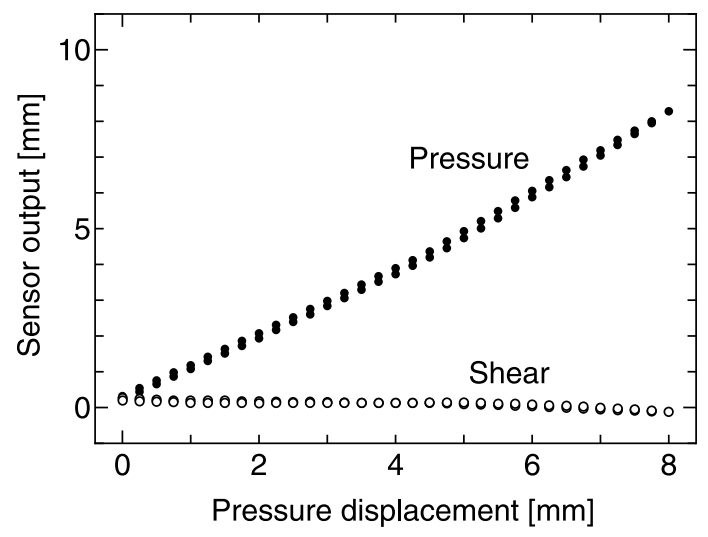

Fig. 9 Sensor outputs under pressure

\subsection{Spring sheared to $90^{\circ}$-direction}

Finally, the spring is sheared to the direction of $90^{\circ}$ compared with previous section. The data in cases of compression and shearing are shown in Fig. 11.

It can be confirmed that the shearing signal in this case is approximately zero and this sensor has the selectiveness of the shear direction. The compressing signal is affected by shear more than the one in the previous section. The moments in the case of shear to the $90^{\circ}$-direction are written as follows:

$$
\begin{aligned}
& M_{1}=-P R+Q H, \\
& M_{2}=0, \\
& M_{3}=0 .
\end{aligned}
$$

Therefore, not only compression force, $P$, but also shearing force, $Q$, is shown as the torsional moment, $M_{1}$. However, the influence can be easily reduced by attaching an additional gage, detecting the shear in this direction and calibrating with three variables $(x, y, z)$.

\section{Conclusions}

In this paper, the structure of the helical spring was focused on and a pressure and shear sensor using it was proposed. The structure of a helical spring translates compressive and shearing forces of spring to torsional and bending moments of wire. The stresses and strains of the spring wire were considered and the guideline for the appropriate angle of gages was derived. The calibration procedure using least square approximation was shown. Finally, experiments were carried out and the effectiveness was confirmed. 


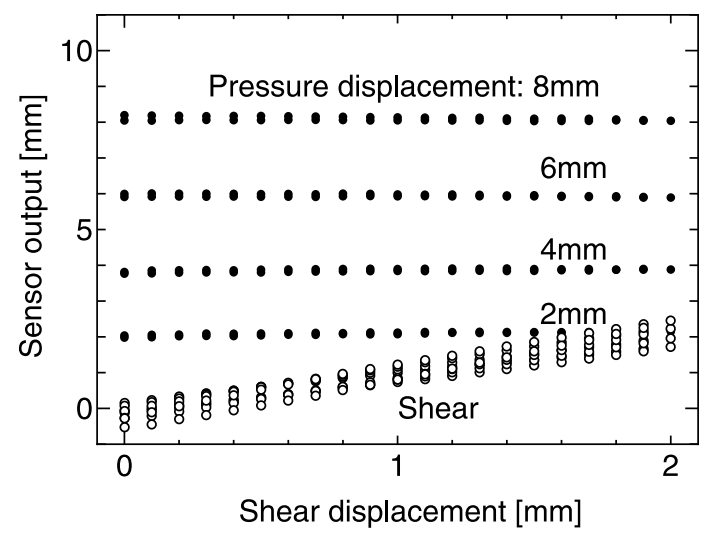

Fig. 10 Sensor outputs under shear

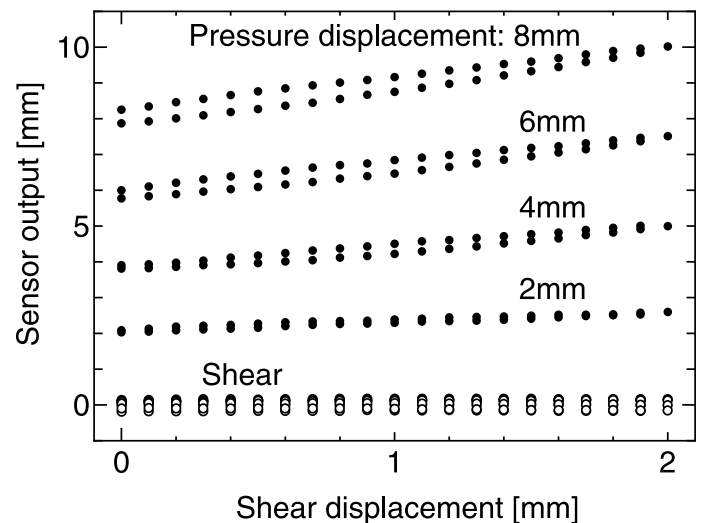

Fig. 11 Sensor outputs under shear and pressure in case of $90^{\circ}$-direction

\section{Acknowledgment}

A part of the present study was supported by MEXT Grant-in-Aid for Scientific Research (B) (No.15300056).

\section{References}

(1) S. C. Cheng, T. Kawai and T. Maeno, Detection of Friction Coefficient using Strain Distribution of Semicircular Elastic Finger Proc. of JSME 1999 Annual Meeting (V), (1999), pp.235-236 (in Japanese).

( 2 ) S. Sasaki, W. H. Sheong, K. Nakamura and H. Shinoda, Instantaneous Detection of the Friction Coefficient Using ARTC Tactile Sensor, Proc. of SICE'99, (1999), pp.339-340 (in Japanese).

( 3 ) Y. Yamada, H. Morita and Y. Umetani, A Slip Sensor with Surface Ridges Which is Mounted on a Robot Hand Generates High-Frequency Signals for Isolating Slip Phases, Trans. of SICE, Vol.36, No.6 (2000), pp.473-480 (in Japanese).

( 4 ) T. Nara, T. Maeda, S. Ando and S. Tachi, A Theory of Skin's Orthogonal Deformation Detected by Mechanoreceptors, Proc. of BPES 2000, (2000), pp.407-410 (in Japanese).

( 5 ) A. Sano, K. Nishi, H. Miyanishi and H. Fujimoto, Development of Multi-Fingered Hand for Telepresence Based on Tactile Information, Trans. of SICE, Vol.40, No.2 (2004), pp.164-171 (in Japanese).

( 6 ) Japan Society of Spring Engineers, Spring (3rd edition), (1982), Maruzen (in Japanese).

( 7 ) K. Kokubo, Y. Goto, T. Mori and M. Tateno, Strength of Material, (2002), Maruzen (in Japanese).

( 8 ) K. Ogawa, Measurement technology of motion for structures and human body, (2002), Tokyo Denki University Press (in Japanese). 